\title{
Thermal Force on Particles in the Transition Regime
}

\author{
GEORGE S. SPRINGER
}

The University of Michigan, Ann Arbor, Michigan, 48104

Received January 19, 1970; accepted June 9, 1970

\begin{abstract}
An interpolation method is proposed for predicting and correlating thermal forces acting on spherical particles suspended in a rarefied gas. The result obtained is applicable over the entire Knudsen number range from free molecule to continuum conditions, and yields the appropriate limiting expressions in the free molecule and continuum regimes. A comparison was made between the result of the present method and available experimental data, and good agreement was found over a wide range of Knudsen numbers and gas to particle thermal conductivity ratios.
\end{abstract}

\section{INTRODUCTION}

In recent years considerable attention has been given to the problem of thermal forces acting on small particles suspended in a gas. Studies of thermal forces have been motivated by interest in the basic phenomena of thermal diffusion in rarefied gases and by interest in the practical applications of these forces to problems related to air pollution, cloud physics, and particle fallout.

The term thermal force is generally defined as a force, other than that caused by convection, which acts on a body suspended in a gas not in equilibrium (1). It is well known that this force depends strongly upon the Knudsen number $K n$, which is defined as the ratio of the mean free path in the gas to the characteristic dimension of the body. In problems where the thermal force acts on a small spherical particle (radius $a$ ) suspended between two parallel plates a distance $H$ apart ( $a \ll H$, see Fig. 1$)$ there are three characteristic Knudsen numbers (2): one based on particle radius $\left(K n_{a} \equiv \lambda / a\right)$, one on the distance between the plates $\left(K n_{H} \equiv\right.$ $\lambda / H)$, and one on the shortest distance between the center of the particle and the plates $h\left(K n_{h} \equiv \lambda / h\right)$. When $H$ and $h$ are of the same order of magnitude (i.e., the particle is not too close to either one of the plates ) only one of the latter two Knudsen numbers needs to be considered. In order to simplify our considerations and still bring out the essential features of thermal forces in a rarefied gas we shall investigate here such situations and shall select $K n_{H}$ as the Knudsen number which, in addition to $K n_{a}$, characterizes the system.

Ideally, theoretical analysis should describe the thermal force over the entire range of Knudsen numbers, i.e., $0<K n_{a}<\infty$ and $0<K n_{H}<\infty$. Such analyses would have to be based on the Boltzmann equation, but owing to the well-known difficulties involved in obtaining solutions to the Boltzmann equation all analyses proposed thus far are restricted to certain limited ranges of the Knudsen number. The approximate Knudsen number ranges in which the available analytical results are applicable are illustrated in Fig. 2. In this figure the indicated limits of the various density regimes were defined arbitrarily as follows: continuum $K n<0.01$, slip $0.01<K n<0.1$, transition $0.1<K n<10$, and free molecule $K n>10$.

As can be seen from Fig. 2, all existing analyses are restricted to the conditions $(a)$ where both $K n_{n}$ and $K n_{a}$ are much greater than unity (completely free molecule flow) 
or $(b)$ where $K n_{H}$ is much smaller than unity $\left(K n_{H} \rightarrow 0\right)$. In analytic studies this latter condition is generally established by assuming that the gas extends to a large distance from the particle $(H \rightarrow \infty)$. Once $K n_{H}$ is taken to be very small then one needs to consider the particle Knudsen number $K n_{a}$ as an independent parameter with the dependence on $K n_{H}$ an implicit one. However, even for this case there is no single analysis

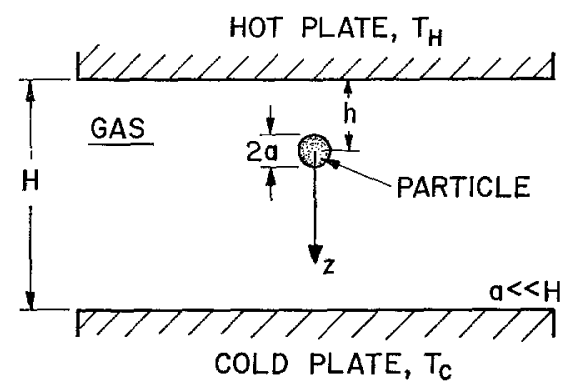

FIG. 1. Description of the problem. that would cover the entire particle Knudsen number range $\left(0<K n_{a}<\infty\right)$.

When $K n_{H} \gg 1$ (and consequently $K n_{a} \gg 1$, since $\left.a \ll H\right)$, the thermal force can be found from simple gas kinetic calculations. For small temperature differences $\left(1-T_{C} / T_{H}<1\right)$ and for complete thermal accommodation at the plates Brock (2) gives the following expression for the thermal force in the $Z$ direction shown in Fig. 1

$$
F=\frac{\pi a^{2}}{4} p_{0} \frac{T_{H}-T_{c}}{T_{0}}, \quad \begin{aligned}
& K n_{a} \gg 1 \\
& K n_{H} \gg 1
\end{aligned}
$$

Here $T_{H}$ and $T_{C}$ are the temperatures of the hot and cold plates, and $p_{0}$ and $T_{0}$ are the mean gas pressure and temperature, respectively. Analytical results are not available for problems where $K n_{H}$ is of the order of unity $\left(K n_{H} \sim 1\right)$. Analytical results have been developed, however, for the case $K n_{H} \ll 1$, and in the following we shall be

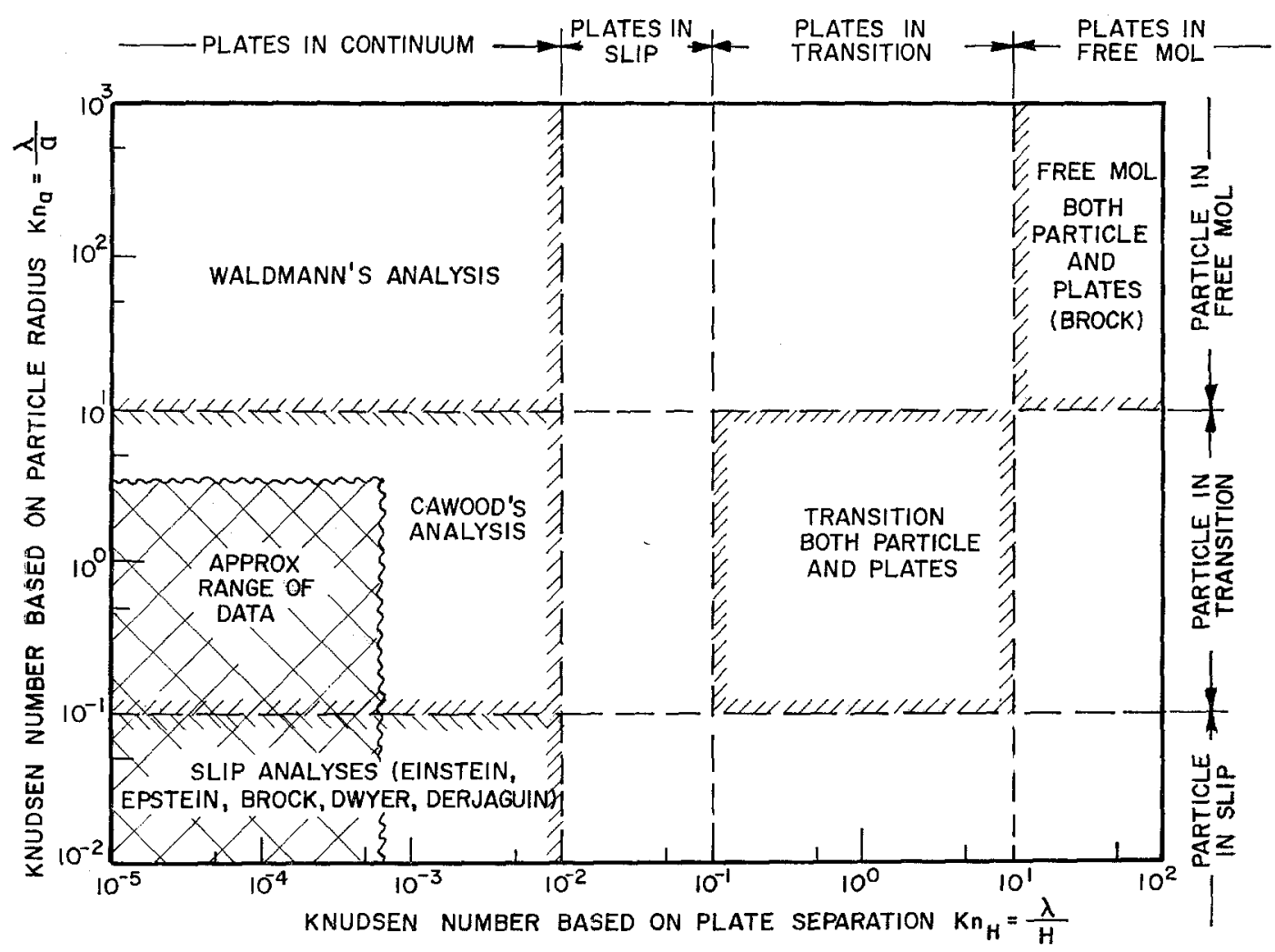

FxG. 2. Knudsen number ranges of the available analytical results and experimental data. 
concerned only with problems where this condition is satisfied. When $K n_{H} \ll 1$ and when the particle is in free molecule flow $\left(K n_{a} \gg 1\right)$, the thermal force can be calculated in a straightforward manner. For a stationary particle Waldmann $(3,4)$ obtained the following expression for the thermal force:

$$
\begin{aligned}
& F_{F M}=-\frac{8 a^{2}}{15}\left(\frac{2 \pi}{R T}\right)^{1 / 2} k_{g} \nabla T_{\infty}, \\
& K n_{a} \gg 1 \text {; } \\
& K n_{H} \ll 1 \text {. }
\end{aligned}
$$

Here $k_{g}$ is the translational gas thermal conductivity, $R$ is the gas constant, $T$ is the mean temperature of the gas at the position of the particle, and $\nabla T_{\infty}$ is the uniform temperature gradient in the gas at a large distance from the particle (i.e., at $H \rightarrow \infty$ ). If we allow $K n_{B} \rightarrow 0$ by letting $\lambda \rightarrow 0$ while keeping $H$ finite, then $\nabla T_{\infty}=\left(T_{H}-T_{C}\right) / \mathrm{H}$. Whereas data have not been obtained yet at Knudsen numbers sufficiently high for a comprehensive evaluation of Waldmann's result (see Fig. 2), Eq. [2] is expected to be accurate at high Knudsen numbers.

The analyses are considerably more complex at the other end of the rarefaction scale, where the mean free path is small compared to the particle radius (slip regime, $K n_{a}<0.1$, say). Approximate analyses for this regime have been presented by Einstein (5), Epstein (6), Brock (7,8), Dwyer (9), and Derjaguin and his coworkers (10-12). Although many experiments have been performed to test these theories (e.g., 1, 8, 1317 ) there is still a lack of firm agreement on the relative accuracies of these theoretical results under various experimental conditions. It has been observed, however, that all these results become increasingly inaccurate as $K n_{a}$ increases.

Less information is available in the transition regime. Here the only analytical results are due to Cawood (18) and Brock (19). Cawood's results fail to describe the thermal force accurately. Brock's approximate analy- sis is expected to be reasonably accurate at near free molecule conditions only $\left(K n_{a}>1\right)$. In the transition regime $\left(0.2<K n_{a}<\infty\right)$, Schmitt (14) found that the data can be correlated by the simple empirical formula $F=F_{F M} e^{-\tau / K n_{a}}$ where $\tau$ is constant, independent of $K n_{a}$, but dependent upon the gas and the particle. This formula was also derived analytically by Brock (19) for the case $K n_{a}>2$. The constant $\tau$ must be determined from experimental data or can be calculated from an expression derived by Brock (19, 20).

It is evident that no simple analytical result is available that would correlate the data or predict the thermal force a priori over the range $0<K n_{a}<\infty$. In this investigation a formula is proposed that correlates well the existing data and which can be used to evaluate the thermal forces on particles over the entire particle Knudsen number range from free molecule $\left(K n_{a} \rightarrow \infty\right)$ to continuum $\left(K n_{a} \rightarrow 0\right)$ conditions, when the Knudsen number based on plate separation is small compared to unity $\left(K n_{H} \ll 1\right)$.

\section{ANALYSIS}

The problem to be considered is the following: a spherical particle of radius $a$ is suspended in a gas contained between two infinite parallel plates. The temperatures of the plates are $T_{H}$ and $T_{C}$, respectively (Fig. 1). The density (or pressure) of the gas is specified and is at such a level that the mean free path in the gas $\lambda$ is very small compared to both distances $H$ and $h(\lambda / H \ll$ 1 , and $\lambda / h \ll 1)$. The ratio $\lambda / a$, however, may have any value from zero to infinity. It is desired to find an expression for the net thermal force acting on the particle.

The expression for the thermal force here derived is based on a method proposed by Sherman (21) for correlating results for heat transfer and shear in rarefied gases and for estimating results when there is insufficient experimental or analytical information to establish a correlation. Sherman's method has been found to be in excellent agreement 
with heat transfer data (22). Therefore, application of this method may be also fruitful in the evaluation of thermal forces in rarefied gases.

In Sherman's method the dependent variable is chosen in such a way that it becomes independent of the Knudsen number as $K n$ becomes large. The independent variable is a quantity proportional to the Knudsen number. For instance, for the normalized thermal force Sherman's interpolation formula can be expressed as follows

$$
F / F_{F M}=\left[1+\left(F_{F M} / F_{C}\right)\right]^{-1},
$$

where $F_{F M}$ is the thermal force in the free molecule limit $(K n \rightarrow \infty)$ and $F_{C}$ is the thermal force at small Knudsen numbers $(K n \ll 1)$. Although it is possible to establish formulas which are more accurate than Eq. [3] (23), the slight increase in accuracy offered by these formulas is offset by the considerable increase in their complexity.

Equation [3] can be applied as long as $F / F_{F M}$ is a monotonic function of the Knudsen number $K n_{a}$, as is the case when $K n_{H}$ is much smaller than unity. ${ }^{1}$ Thus, once $F_{F M}$ and $F_{C}$ are known, the thermal force $F$ can be calculated from Eq. [3] at all particle Knudsen numbers $\left(0<K n_{a}<\infty\right)$, provided that $K n_{H} \ll 1$. It can also be used both with monatomic and diatomic gases. Unfortunately, all the available expressions for $F_{F M}$ and $F_{C}$ are restricted to monatomic gases, and for this reason the analysis that follows will be also limited by this assumption. It is noted here that if the appropriate expressions are selected for $F_{F M}$ and $F_{C}$ then Eq. [3] is applicable both to stationary and to moving particles. In the following we shall be concerned only with stationary particles. However, the method of solution is suffi-

\footnotetext{
1 Note that as long as $K n_{H} \ll 1$ the thermal force varies inversely with pressure. This may not be the case, however, when this condition is not satisfied. For example at $K n_{H} \gg 1$ and $K n_{a} \gg 1$ the thermal force is directly proportional to pressure (see Eq. [1]).
}

ciently general that it could be extended readily to moving particles.

For $F_{F M}$ one may use Waldmann's result given by Eq. [2]. For $F_{C}$ one may select any one of the expressions presented in references 5-12. We arbitrarily select for $F_{C}$ the following expression derived by Jacobsen and Brock (8):

$$
\begin{aligned}
& F_{c}=-\frac{9 \pi \mu^{2} a}{\rho T} \\
& \left\{\begin{array}{c}
\left(k_{g} / k_{p}\right)+C_{t}(\lambda / a) \\
+(4 b / 3) C_{m}(\lambda / a)\left[\left(k_{g} / k_{p}\right)\right. \\
\left.+C_{t}(\lambda / a)-1\right] \\
\frac{1+2\left(k_{g} / k_{p}\right)+(\lambda / a)\left[2 C_{t}\right.}{\left.1+3 C_{m}+6 C_{m}\left(k_{g} / k_{p}\right)\right]} \\
+6 C_{m} C_{t}(\lambda / a)^{2}
\end{array}\right\} \\
& K n_{a}<1 ; \\
& K n_{H} \ll 1 \text {. }
\end{aligned}
$$

In Eq. [4] $\mu$ is the viscosity, $\rho$ the density, $T$ the temperature of the gas, $k_{p}$ is the thermal conductivity of the particle, $b$ is a constant, and $C_{m}$ and $C_{t}$ are constants associated with the slip and temperature jump coefficients, respectively. For monatomic gases $C_{\ell}$ and $C_{m}$ are related to the thermal accomodation coefficient $\alpha$ and the tangential momentum accommodation coefficient $\sigma$ by $(7,24)$

$$
C_{t} \cong \frac{15}{8} \frac{2-\alpha}{\alpha} \text { and } C_{m} \cong \frac{2-\sigma}{\sigma} .
$$

By setting $b=2.4$, as suggested by Jacobsen and Brock (8), and by using the relationships $\mu=0.5(8 R T / \pi)^{1 / 2}(\lambda \rho)$ and $k_{g}=$ $(15 / 4) R \mu$, Eqs. [4] and [5] can be rearranged to yield

$$
\frac{F}{F_{F M}}=\left\{1+\frac{2}{9} \frac{\lambda}{a}\right.
$$




$$
\left.\begin{array}{r}
1+\frac{2 k_{g}}{k_{p}}+\frac{\lambda}{a}\left[2 C_{t}\right. \\
\left.+3 C_{m}\left(1+\frac{2 k_{g}}{k_{p}}+2 C_{t} \frac{\lambda}{a}\right)\right] \\
\frac{k_{g}}{k_{p}}+\frac{\lambda}{a} C_{t} \\
+3.2 C_{m} \frac{\lambda}{a}\left(\frac{k_{g}}{k_{p}}+C_{t} \frac{\lambda}{a}-1\right) \\
0 \leqq K n_{a} \leqq \infty ; \\
K n_{H} \ll 1 .
\end{array}\right\}
$$

[6] calculated from Eq. [6] over the Knudsen number range $0<K n_{a}<\infty$. In order to check the accuracy of this equation, thermal forces calculated from $\mathrm{Eq}$. [6] were compared to thermal forces measured by Jacobsen and Brock (8), by Schadt and Cadle (16), and by Waldmann $(3,4)$. It is noted here that the data reported by Waldmann are the same as given by Schmitt (14). These experimental data were obtained for a wide range of gas to particle thermal conductivity ratios $\left(k_{g} / k_{p}=0.0022-0.133\right)$ and for a wide range of Knudsen numbers $\left(K n_{a}=0.05-4.0\right)$.

In order to make the comparison shown in Fig. 3, one must select values for $C_{m}$ and $C_{t}$. For most engineering surfaces the thermal accommodation coefficient $\sigma$ is between 0.7 and $1.0(26,27)$. For their data Jacobsen and Brock suggest the value of 0.72 for $\alpha$. Very little information exists on the tangential accommodation coefficient $\sigma$, but the available data indicate that $\sigma$ is very close to unity (26). Therefore, here the values $\alpha=0.72$ and $\sigma=1.0$ were selected, giving $C_{t}=3.32$ and $C_{m}=1.0$ (see Eq. [5]).

Given the properties of the gas and the particle, the parameters $C_{m}$ and $C_{t}$, and the imposed temperature field $\nabla T_{\infty}$, the thermal force acting on a particle of radius $a$ can be

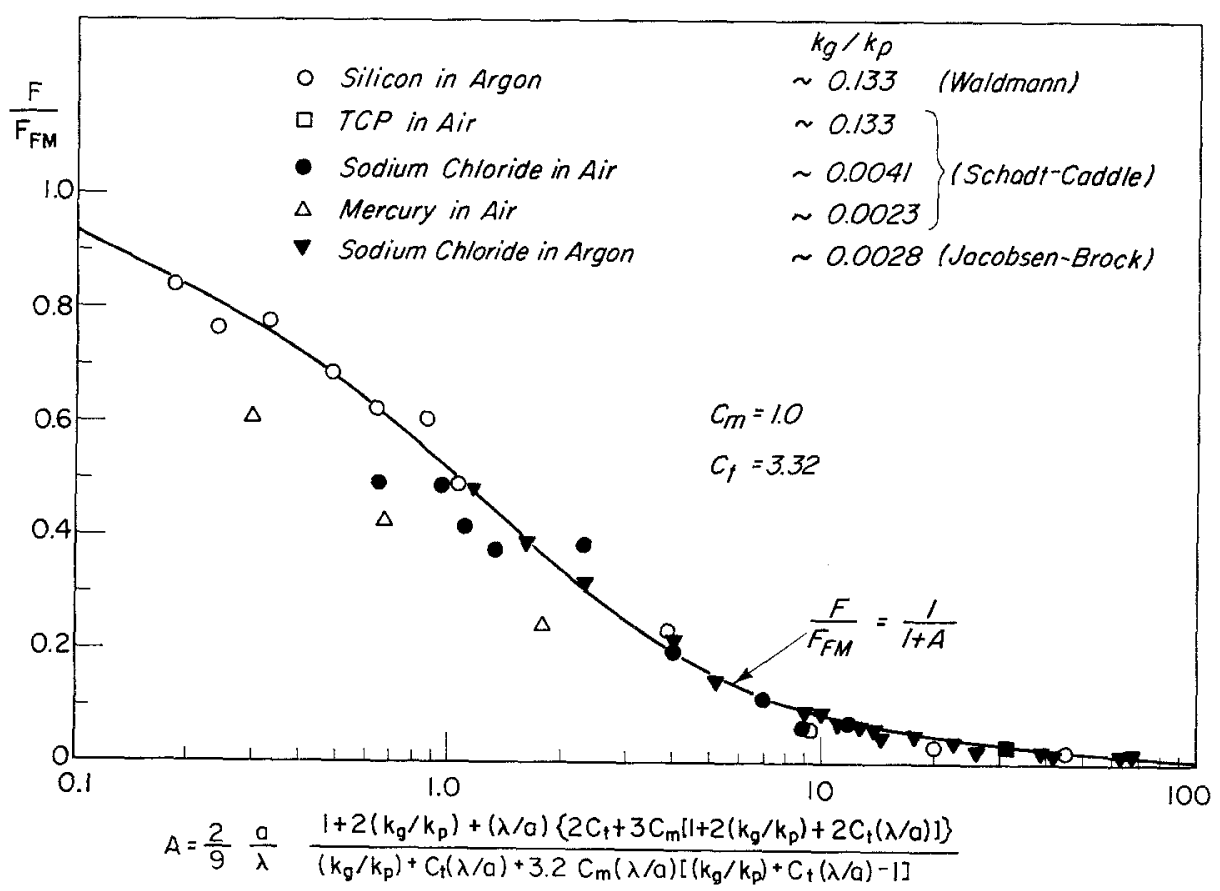

Frg. 3. Comparison between the experimental data and the formula proposed in this investigation (Eq. [6]). 
The comparison given in Fig. 3 shows good agreement between the results of the formula here proposed (Eq. [6]) and the data over the entire range of the parameters tested. Interestingly, Eq. [6] agrees with the data well even for those experiments where a diatomic gas (air) was used. It appears, therefore, that $\mathrm{Fq}$. [6] can be used to calculate the thermal force with reasonable accuracy, and lacking more accurate information on the parameters $C_{m}$ and $C_{t}$, the values $C_{m}=1.0$ and $C_{t}=3.32$ are reasonable for use in the calculations. The only exception to this appear to be the data for mercury, which fall somewhat below the theoretical curve. A higher $\alpha$ value (and consequently a higher $C_{\imath}$ value) would give better agreement between the theoretical results and the mercury data, suggesting that for the mercury-air combination $\alpha$ was close to unity.

Finally, it is pointed out that although Eq. [6] is restricted to problems where $K n_{H}$ $\ll 1$, it can be used to correlate all the experimental data reported in the literature. This is demonstrated in Fig. 2, where the Knudsen number ranges covered by the existing data are shown. From this figure it can be seen readily that although experiments have been performed at particle Knudsen numbers corresponding to the slip and transition regimes $\left(0.01<K n_{a}<10\right)$, the measurements extend only to $K n_{H} \sim 5 \times 10^{-3}$. The wide Knudsen number ranges where data are still lacking and where further measurements would be desirable are evident from Fig. 2.

\section{ACKNOWLEDGMENT}

This work was supported by the United States Atomic Energy Commission.

\section{REFERENCES}

1. Rosenblatt, P., and Lamer, V. K., Phys. Rev. 70, 385 (1946).
2. Brock, J. R., J. Colloid and Interface Sci. 25, 564 (1967).

3. Waldmann, L., Z. Naturforsch., A 14, 589 (1959).

4. Waldmann, L., in L. Talbot, Ed., "Rarefied Gas Dynamics," p. 323. Academic Press, New York, 1961.

5. Einstein, A., Z. Phys. 27, 1 (1924).

6. Epstein, P. S., Z. Phys. 54, 537 (1929).

7. Brock, J. R., J. Colloid Sci. 17, 768 (1962).

8. J ACobsen, S., And Brock, J. R., J. Colloid Sci. 20, 544 (1965).

9. Dwyer, H. A., Phys. Fluids 10, 976 (1967).

10. Derjaguin, B. V., and Bakanov, S. P., Dokl. Akad. Nauk SSSR 141, 384 (1961).

11. Deruaguin, B. V., and Bakanov, S. P., Dokl. Akad. Nauk SSSR 147, 139 (1962).

12. Deruaguin, B. V., and Yalamov, Y. I., Dokl. Akad. Nauk SSSR 155, 886 (1964).

13. Saxton, R. L., and Ranz, W. E., J. Appl. Phys. 23, 917 (1952).

14. Sснмiт, K. H., $Z$. Naturforsch, $A$ 14, 870 (1959).

15. Schadt, C. F., and Cadle, R. D., J. Colloid Sci. 12, 356 (1957).

16. Schadt, C. F., And Cadle, R. D., J. Phys. Chem. 65, 1689 (1961).

17. Derjaguin, B. V., Storozhilova, A. I., ANd Y. I. Rabinovich, J. Colloid Interface Sci. 21, 35 (1966).

18. Cawood, W., Trans. Faraday Soc. 32, 1068 (1936).

19. Вrock, J. R., J. Colloid Interface Sci. 23, 448 (1967).

20. Brock, J. R., J. Colloid Interface Sci. 25, 392 (1967).

21. Sherman, F. S., in J. A. Lauermann, Ed., "Rarefied Gas Dynamics," Vol. 2, p. 228. Academic Press, New York, 1963.

22. Springer, G. S., Fluid Dynamics Laboratory Report No. 69-1, Dept. of Mech. Eng., The Univ. of Michigan, Ann Arbor, 1969.

23. Wiulis, D. R., Amer. Soc. Mech. Engrs. Paper 69-WA/HT (1969).

24. Kennard, E. H., "Kinetic Theory of Gases," pp. 295-315. McGraw Hill, New York, 1938.

25. Wachman, H. Y., J. Amer. Rocket Soc. 32, 2 (1962).

26. SchaA F, S. A., in W. H. T. Loh, Ed., "Modern Developments in Gas Dynamics," p. 235. Plenum Publishing Corp., New York, 1969. 